\title{
Long term transvenous ventricular pacing in adults with congenital abnormalities of the heart and great arteries
}

\author{
D E WARD, B CLARKE, P M SCHOFIELD, SUE JONES, K DAWKINS, D BENNETT \\ From the Cardiology Departments, Wythenshawe Hospital, Manchester, and the Brompton Hospital, London
}

SUMMARY Improved techniques of transvenous pacing have made it possible to use this approach in various congenital cardiac abnormalities. Transvenous active fixation pacing leads were implanted in seven patients aged 15 to 81 years with abnormal venous, atrioventricular, or ventriculoarterial connections with successful ventricular pacing.

For long term pacing the transvenous approach is now widely preferred to the epicardial method because it is more convenient and associated with fewer complications. ${ }^{1}$ The recent availability of a range of active fixation electrodes has resulted in a fall in the displacement rate ${ }^{2}$ with a wider choice of pacing sites. ${ }^{3}$ The stability of this type of endocardial electrode has made it possible to undertake transvenous pacing in adults $^{4}$ and children ${ }^{5}$ with cardiac anomalies. We report the use of active fixation electrodes in seven adults (Table) with congenital abnormalities of the heart which would have made conventional pacing methods difficult or impossible.

\section{Case reports}

\section{CASE 1}

A 22 year old man was found to have complete transposition of the great arteries with an associated ventricular septal defect at the age of 7 weeks. He underwent a Blalock procedure at five years of age, Mustard operation and repair of the defect at 13 years, and a revision three years later. After surgery he developed atrial tachycardias and atrioventricular block. An epicardial pacemaker was implanted. This system failed because of exit block, and an endocardial approach was attempted. A Helifix (Vitatron) lead was introduced via the right subclavian vein, manipulated past the interatrial baffle, and positioned in the posterior morphologically left (venous) ventricle (Fig. 1). During follow up for 10 months there has been stable pacing.

Accepted for publication 22 June 1983
CASE 2

This patient was investigated shortly after birth and found to have a single ventricle associated with absent right atrioventricular connection. He was reassessed in 1982 and found to have a univentricular heart of left ventricular type with tricuspid atresia, ventriculoarterial concordance, and pulmonary stenosis. During childhood his electrocardiogram had shown prolongation of the P-R interval and intermittent atrioventricular Wenckebach conduction. In 1978 at the age of 16 years he was in complete atrioventricular block. Two years later he began to experience dizzy spells and permanent pacing was considered to be advisable. A Fontan procedure was also contemplated at this time and he was admitted for measurement of the pulmonary artery pressure. A permanent pacing system was implanted at the same time. A Helifix lead was introduced via the right subclavian vein and positioned in a posteriorly situated morphologically left ventricle. It was thought that the electrode had passed into the left ventricle via the atrial septal defect. Stable pacing has been maintained during the 15 months of follow up. At operation it was apparent that the transvenous electrode had traversed a small stenotic right atrioventricular valve and passed into the morphological left ventricle via a small ventricular septal defect. Thus, this patient had a univentricular heart of left ventricular type with double inlet and ventriculoarterial concordance with a restrictive defect causing effective pulmonary stenosis (Holmes's heart). The right atrioventricular valve was hypoplastic and there was a large atrial septal defect. No corrective or reparative surgery was performed. 
Table Clinical data and transvenous pacing system used in seven patients with congenital cardiac abnormalities

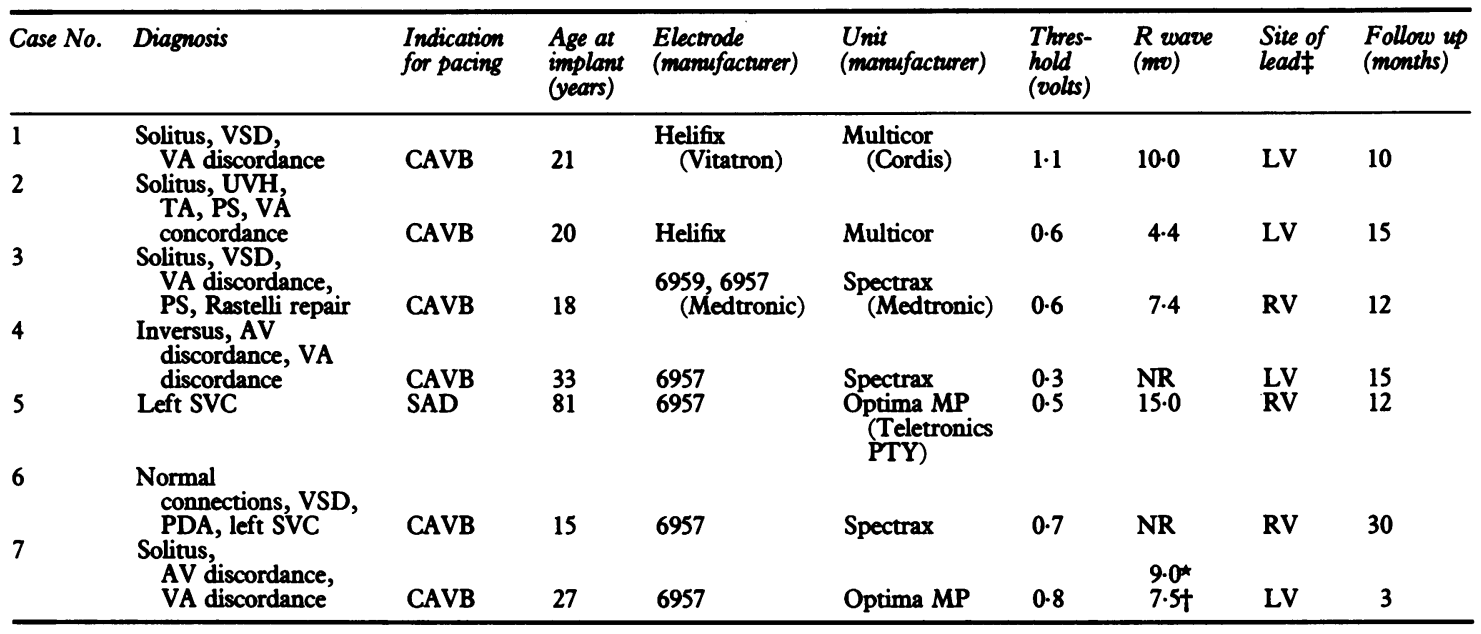

^First procedure.

tAt revision.

fMorphological description.

VSD, ventricular septal defect; VA, ventriculoarterial; AV, atrioventricular; PS, pulmonary stenosis; SVC, superior vena cava; PDA, persistent ductus arteriosus; CAVB, complete atrioventricular block; SAD, sinuatrial disorder; LV, left ventricle, RV, right ventricle; UVH, univentricular heart; TA, tricuspid atresia; NR, not recorded.

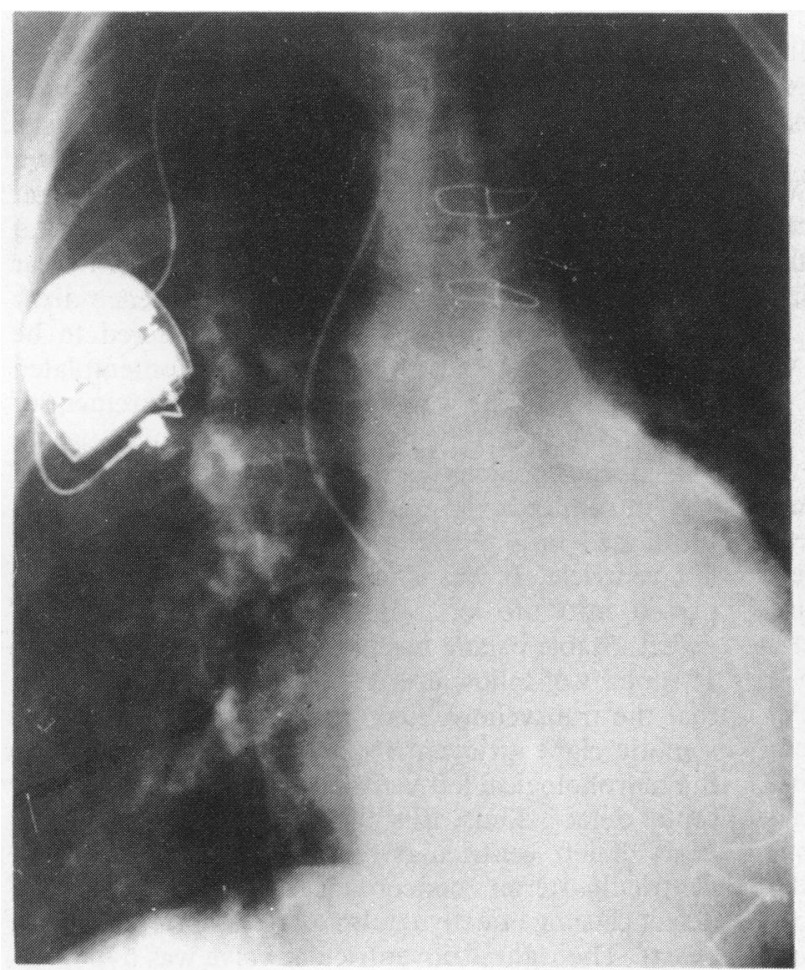

(a)

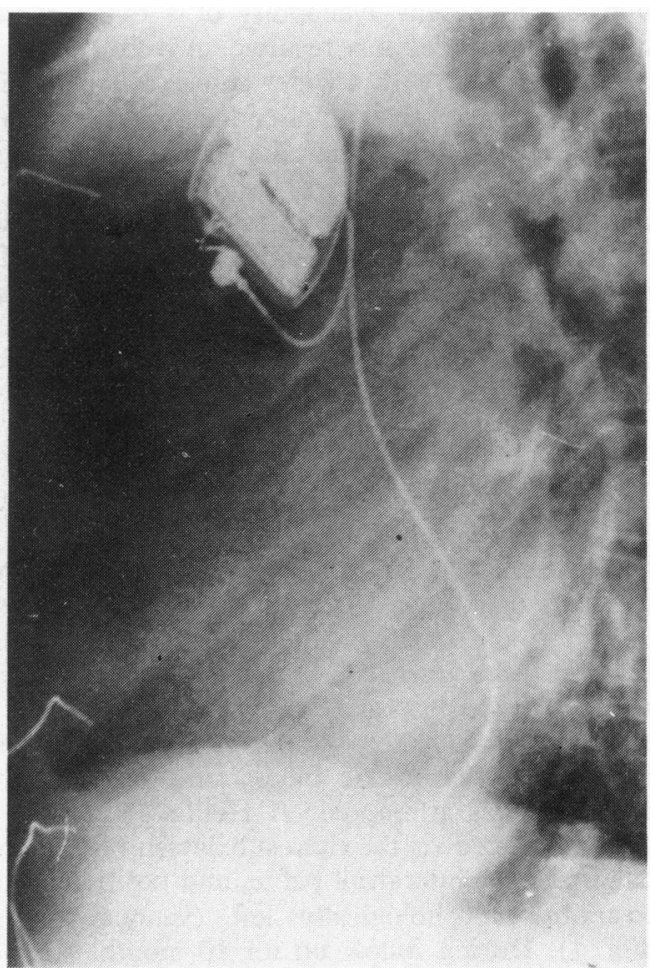

(b)

Fig. 1 Posteroanterior (a) and lateral (b) chest radiographs showing endocardial pacing system (case 1). The electrode passes around the interatrial baffle (Mustard operation) and into the posterior morphologically left ventricle which gives rise to the pulmonary artery. 


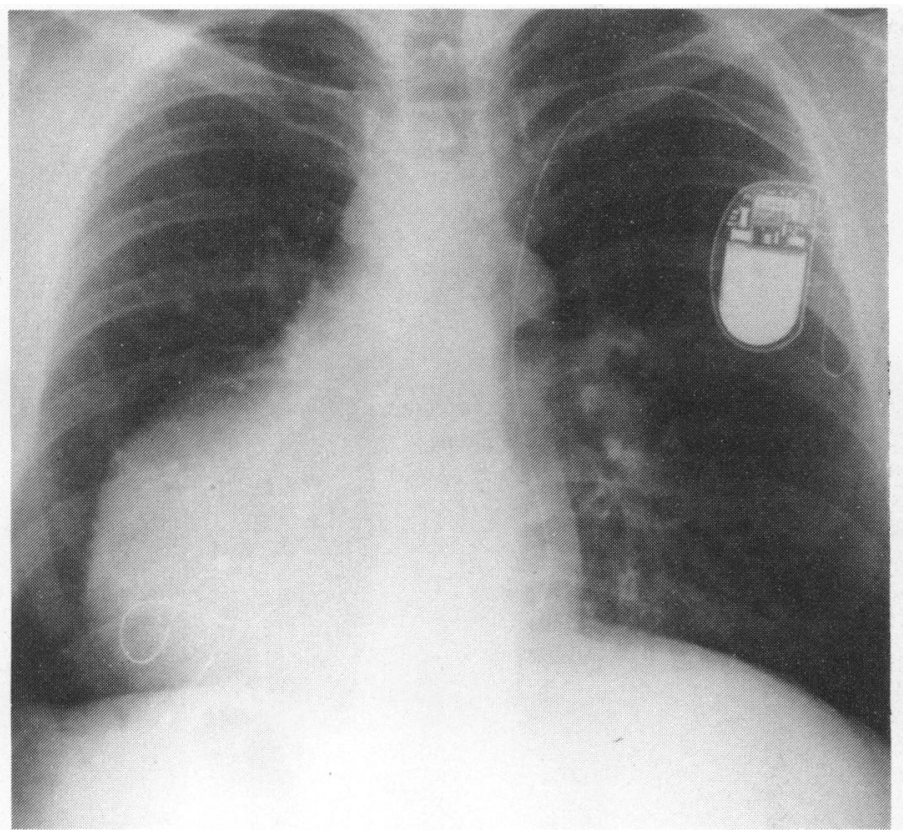

Fig. 2 Chest radiograph (case 4) showing the pacing electrode in the morphologically left ventricle in situs inversus.

\section{CASE 3}

This patient was noted to be cyanosed at 1 month of age. Catheterisation showed complete transposition with infundibular pulmonary stenosis, a ventricular septal defect, and a patent foramen ovale. At the age of 13 years a Rastelli procedure was performed after which he developed complete atrioventricular block. An epicardial pacing system was implanted but eroded through the skin after one year and was revised. In 1981 an endocardial system was inserted. A Medtronic 6959 screw in electrode was inserted via the left cephalic vein and positioned in the right ventricle. Care was taken to obtain satisfactory endocardial electrocardiograms before advancing the screw, thus ensuring that the prosthetic material used in the repair was avoided. The system was revised because of exit block a year later. A similar approach was used and proved to be entirely successful. Since then there have been no further pacing problems. A different electrode was used at revision because the 6959 had been withdrawn by the manufacturers for modification.

\section{CASE 4}

This patient presented at 22 years of age complaining of dizziness. The electrocardiogram showed complete atrioventricular block. "Corrected transposition" was suspected and catheterisation was performed. This showed atrioventricular and ventriculoarterial discordance associated with situs inversus. An attempt at transvenous atrial pacing failed after two years and the pacing system was removed. An epicardial system was implanted in 1978 but this failed to capture after one year. The patient refused further operations. Subsequently he suffered a blackout and was admitted to hospital when elective permanent transvenous pacing was undertaken. A Medtronic 6957 lead was inserted via the left subclavian vein and positioned in the apex of the morphologically left ventricle (Fig. 2). Diaphragmatic stimulation occurred in the postoperative period but was controlled by reducing the output of the generator. Stable pacing has been maintained over 12 months' follow up.

\section{CASE 5}

A 78 year old man presented with syncope associated with profound sinus bradycardia. An epicardial pacing system was implanted in May 1980. This functioned for two years when dizziness and blackouts occurred. Testing showed profound myopotential suppression. An endocardial system was implanted using a Medtronic 6957 lead inserted via the left cephalic vein. The lead passed down a persistent left superior vena cava through a dilated coronary sinus into the right atrium. The electrode was easily manipulated into the right ventricle and secured at the apex. There have been no problems during follow up over 12 months.

\section{CASE 6}

This patient presented with clinical evidence of a large left to right shunt at the age of 8 weeks. Cardiac 


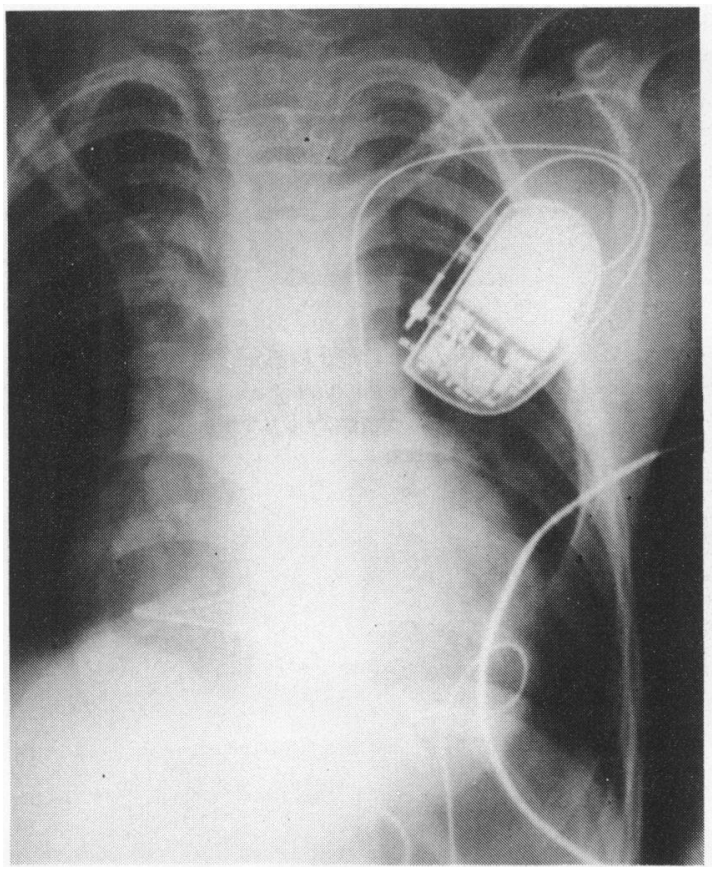

catheterisation showed a ventricular septal defect and a persistent ductus arteriosus. Division of the ductus and banding of the pulmonary artery were performed. At 8 years of age the pulmonary artery was debanded and the defect closed. After this operation he developed complete atrioventricular block. An epicardial pacing system was inserted but failed after two years. A second system was implanted and provided stable pacing until battery failure after four years. An endocardial Medtronic 6957 lead was implanted electively via a left superior vena cava (Fig. 3). This case has been reported in detail elsewhere. ${ }^{4}$

\section{CASE 7}

A 27 year old patient was diagnosed as having situs solitus and atrioventricular and ventriculoarterial discordance at the age of 8 years. At that time she was in complete atrioventricular block. Nevertheless, she remained well until the age of 27 years when she had a single episode of loss of consciousness, typical of an Adams-Stokes attack. A permanent endocardial electrode (Medtronic 6957) was inserted via the left subclavian vein into a posteriorly located morphologically left ventricle. Postoperative recovery was complicated by left shoulder pain and diaphragmatic stimulation, probably the result of perforation of the left ventricle by the screw. Echocardiography showed no pericar-
Fig. 3 Chest radiograph (case 6) showing passage of electrode in left superior vena cava.

dial effusion and her symptoms responded to mild analgesics. The output of the unit was reprogrammed from 5.0 to 2.5 volts and diaphragmatic stimulation ceased. Unfortunately, diaphragmatic stimulation returned after several weeks and resiting of the electrode was undertaken three and a half months after the initial implant. She has now been followed for two months without further problems.

A satisfactory endocardial pacing threshold was obtained in all patients (Table). In the five patients in whom an endocardial $\mathbf{R}$ wave potential was obtained, this was satisfactory in all. Multiprogrammable pacemakers were used in each case to provide greater flexibility during surveillance.

\section{Comment}

The indication for permanent pacing in our patients was complete atrioventricular block in six (in three this rhythm was a complication of a surgical procedure) and sinuatrial disease in one. In four patients epicardial systems had been used previously, and in each these systems had caused problems. For this reason, or because of previous cardiac surgery, we decided to attempt transvenous pacing electively using active fixation electrodes. There is little doubt that the incidence of dislocation of endocardial leads has been considerably reduced by the use of these 
electrodes. ${ }^{2}$ In addition, long term pacing at unconventional sites is possible with certain types of active fixation electrode. ${ }^{3}$ In children large loops may be fashioned to allow for growth. ${ }^{5}$. This could cause instability of passive fixation (tined) or non-fixation leads. These findings coupled with improved venous access $^{6}$ led us to believe that long term endocardial pacing could be achieved in selected patients with congenital abnormalities of venous, atrioventricular, and ventriculoarterial connections.

The advantages of active fixation electrodes for endocardial pacing via a persistent left superior vena cava have been emphasised previously. ${ }^{4}$ In both patients with this anomaly reported here epicardial pacing systems had been unsuccessful. In the five other patients endocardial pacing was possible despite abnormal atrioventricular or ventriculoarterial connections. In the patient with a Rastelli repair an active fixation lead was chosen to allow greater choice of endocardial sites in the presence of a considerable amount of prosthetic material. The electrode was sited in the morphologically left ventricle in four of our patients. It is likely that conventional electrodes would be unsuitable for long term pacing in this relatively smooth walled chamber because of poor stability. In three of these patients the left ventricle was the venous ventricle but in one (case 2 ) it gave rise to the aorta. There is, therefore, a small risk of systemic emboli in this patient but there has been no clinical evidence of this. Where appropriate, however, long term anticoagulant treatment is a reasonable precaution. In two patients (cases 4 and 7) in whom the pacing electrode was inserted into a morphologically left ventricle diaphragmatic stimulation occurred which ceased when the output of the pacemaker was reduced. It seems likely that in this situation penetration of the myocardium may occur because the ventricle is smooth and thin walled without trabeculation. If this is recognised at operation resiting the electrode to another area should avoid this problem.

The advantages of endocardial pacing over epicardial pacing in the presence of congenital cardiac abnormalities which have required previous surgery have been emphasised by McGoon et al. ${ }^{7}$ They described four patients with complex congenital heart disease in whom atrial pacing was undertaken to control sinus bradycardia and atrial arrhythmias. This approach would have been inappropriate in six of our patients, however, because the cause of the bradycardia was atrioventricular block.

In conclusion, transvenous endocardial pacing in adult patients with congenital cardiac abnormalities is made possible by recent advances in permanent electrode design and improved methods of implantation. Even in the presence of complex lesions it may be possible to locate the electrode at a stable endocardial site. Transvenous pacing is preferrable to epicardial pacing because thoracotomy is avoided. This consideration is especially important in patients who have had one or several previous cardiac operations which may make further surgery technically difficult. In patients with complex anomalies, angiographic or echocardiographic delineation of the anatomical defects is essential before permanent endocardial pacing, whether atrial or ventricular, is attempted.

\section{References}

1 Oldershaw PJ, Sutton MG, Ward D, Jones S, Miller GAH. Ten year experience of 359 epicardial pacemaker systems: complications and results. Clin Cardiol 1982; 5: 515-9.

2 Bisping HJ, Kreuzer J, Birkenheier H. Three-year clinical experience with a new endocardial screw-in lead with introduction protection for use in the atrium and ventricle. Pacing and Clinical Electrophysiology 1980; 3: 424-5.

3 Nathan AW, Ward DE, Hellestrand KJ, Jones SM, Giles IL, Camm AJ. The right ventricular outflow tract-an alternative permanent pacing site [Abstract]. PACE 1982; 5: 301.

4 Hellestrand KJ, Ward DE, Bexton RS, Camm AJ. The use of active fixation electrodes for permanent endocardial pacing via a left superior vena cava. PACE 1982; 5: $180-4$.

5 Ward DE, Signy M, Oldershaw P, Jones S, Shinebourne EA. Cardiac pacing in children. Arch Dis Child 1982; 57: $514-20$.

$6 \mathrm{McNeill} \mathrm{GP,} \mathrm{Taylor} \mathrm{NC.} \mathrm{Use} \mathrm{of} \mathrm{subclavian} \mathrm{vein} \mathrm{for} \mathrm{per-}$ manent cardiac pacing. Br Heart $f$ 1978; 40: 114-6.

7 McGoon MD, Maloney JD, McGoon DC, Danielson GK. Long-term endocardial atrial pacing in children with postoperative bradycardia tachycardia syndrome and limited ventricular access. Am $\mathcal{f}$ Cardiol 1982; 49: $1750-7$.

Requests for reprints to David E Ward, Cardiology Department, St George's Hospital, London SW 17 OQT. 